\title{
Evaluating a Web-Based Information System for Managing Master of Science Summer Projects
}

\author{
Till Rebenich \\ University of Southampton \\ United Kingdom \\ tr08r@ecs.soton.ac.uk
}

\author{
Andrew M. Gravell \\ University of Southampton \\ United Kingdom \\ amg@ecs.soton.ac.uk
}

\author{
Thanassis Tiropanis \\ University of Southampton \\ United Kingdom \\ tt2@ecs.soton.ac.uk
}

\begin{abstract}
We describe the design of a web-based information system for monitoring MSc summer projects in the School of Electronics and Computer Science at the University of Southampton, and a mixed method quasi-experimental study involving 290 MSc project students, 19 monitors, and 69 supervisors in electronics and computer science, using the system over a period of 17 weeks. Statistically significant results presented here are: Students making heavy use of the system achieved higher marks on their project dissertation, while no such correlation was found with marks for other parts of their MSc. Likewise, student's monitor activity is significantly correlated with their own activity and dissertation mark. These results suggest that educational information and project management systems positively affect student achievement and academic staff involvement is crucial for these systems to be successful. Future work includes a more detailed analysis of success factors and their impact on student performance.
\end{abstract}

\section{Categories and Subject Descriptors}

H.4 [Information Systems Applications]: Miscellaneous

\section{General Terms}

Design, Experimentation, Human Factors

\section{Keywords}

information systems, e-learning, project management, time management, impact study

\section{INTRODUCTION}

At the University of Southampton and at many other UK universities, the Master of Science (MSc) degree typically takes one calendar year to complete and is concluded by a three-month summer project during which students are expected to do independent research and practical work on

Permission to make digital or hard copies of all or part of this work for personal or classroom use is granted without fee provided that copies are not made or distributed for profit or commercial advantage and that copies bear this notice and the full citation on the first page. To copy otherwise, to republish, to post on servers or to redistribute to lists, requires prior specific permission and/or a fee.

ITiCSE'11 June 27-29, 2011, Darmstadt, Germany

Copyright 2011 ACM 978-1-4503-0697-3/11/06 ...\$10.00. a well-defined topic. At the end of the project, students must submit a dissertation which is marked independently by two or three examiners.

Normally, MSc students pursue their project under the supervision of at least one academic in the school. This concept is very much centred around project-based learning as students are required to work autonomously on a given task and are responsible for investigating a problem, making decisions, planning their work, and coming up with suitable solutions [9]. Due to the limited time available for this part of the MSc, good time management and project planning are crucial success factors.

In 2009/10, the school's MSc student intake significantly exceeded that of previous years. Numbers increased by $73.1 \%$, from 171 in 2008 to 296 in 2009, posing additional challenges to academic staff. Our work focusses on organisational aspects of MSc project management, that is, on supporting project planning and monitoring using information technology. For this purpose, a web-based information system was developed and rolled out in the school in 2010. We did not use off-the-shelf (OTS) software since it either did not meet our requirements or was too difficult to customise and integrate. The system was used in a quasi-experimental mixed method study and served as a prototype for evaluating how similar systems can enhance teaching as part of a virtual learning environment.

The system is aimed at supporting project students in organising their work, reporting their progress, and planning meetings with their supervisor. Additionally, a monitoring scheme was introduced whereby each project student was allocated to a monitoring group led by a postgraduate research student. In total there were 39 groups, and each of them contained 6 students on average. Members of a group were told to meet once a week, raise problems, ask questions, and discuss their progress with peers typically working in the same topic area. These meetings do not replace supervision meetings but are a complementary measure to further support students. The information system supports this scheme by providing features for managing group meetings, interacting with its members, and recording meeting attendance. System use was compulsory for monitors, while supervisors were not required to use it in view of their high workload. It was expected that monitors spend approximately 1 hour per week on filing progress reports, subject to group size, and students could submit their report and manage their project tasks in no more than 30 minutes per week.

Both our system and the monitoring scheme are based on 


\section{Dashboard}

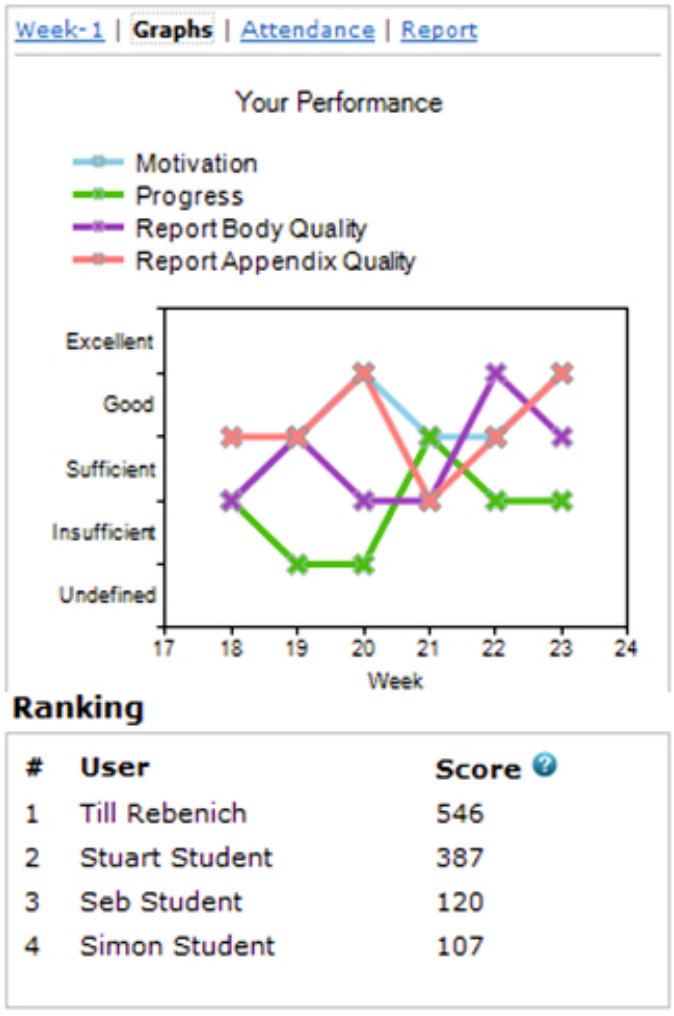

Figure 1: Example of statistics dashboard and student ranking table

a small trial carried out in the summer of 2009 involving only two monitors and a subset of all MSc students. The outcomes of this trial were positive, but the monitors recommended the development of an information system supporting their work.

We also adopted some of the features used in previous work $[8,7]$ in the area of student time management. The positive relationship between good time management skills and academic performance has been emphasised in related work $[1,4]$. In addition, we provided students with features increasing their progress awareness and motivation [5] by exposing certain progress statistics to the whole cohort, enabling students to compare their own progress with that of their peers (see Figure 1). Project monitors also had to report on the progress of each student and record their meeting attendance on the system.

\section{SYSTEM DESIGN}

The web-based information system used here was developed using ASP.NET MVC 2.0 and deployed on a virtual machine inside the school. It was released for general use on 14 June 2010 , and workshops were provided to monitors and interested academic staff. It was also presented and explained to students as part of their project kick-off event. The current version is a stand-alone application available to all computer science and electronics MSc students. Users can log on using their school username and password. It consists of 5 components which are described in more detail in the following sections. They are: project management, meeting and event organisation, progress tracking, meeting attendance monitoring, and communication. To raise students' awareness of ongoing events, feedback submissions, and the activity of other users, email reminders were sent by the system.

Students can create lists of tasks with their interdependencies and prerequisites. For each task, basic metrics such as due date, planned duration, and current progress can be defined. Furthermore, four milestone tasks were pre-defined: submit dissertation brief, end practical work, submit first draft, and submit final report. Regular tasks can be assigned to one of these milestones. All task data can be exported and used with other project management client software such as Microsoft Project.

The system is based on a hierarchical structure of organisational units, namely degree, programme, group, and project. In this structure, the degree is the top-most unit, while a student project is a leaf unit. Multiple users can be associated with each unit. A project is pre-defined for each student, and each student is member of a monitoring group. Both single and recurring events or meetings can be defined in projects or groups. All students were asked to enter their supervision meetings into the system at the beginning of the summer project, and monitors were in charge of managing monitoring group meetings. All event data can be exported and used with other calendaring software, e.g. Google Calendar and others.

Every week, each student, their monitor, and their supervisor were asked to rate the student's overall progress, their motivation, and the quality of their written work on a scale from 0 (not seen) to 5 (outstanding). Ratings are also subject to an underlying ranking system, so that supervisor ratings take preference over monitor ratings, which again take preference over student ratings. Progress ratings were also aggregated and presented to all system users on graphs and charts, and a ranking table showed the performance of each student against their peers. All participants were able to view these statistics online at any time. Charts provided include (1) histograms showing current and last week's progress ratings for the programme cohort, (2) graphs of progress ratings over time, (3) attendance over time, (4) quantitative and qualitative report metrics over time, and (5) task statistics.

All users were prompted for their meeting attendance feedback, that is, whether students had attended a particular event or not. Meeting attendance statistics were also used for ranking purposes and displayed graphically.

Users could interact on group pages using a thread-based messaging system, whereby members could comment on other users' threads in the same group. Furthermore, email addresses of all users were exposed to associated people.

\section{STUDY DESIGN}

As we did not have direct control over the organisation of MSc projects, we chose a quasi-experimental mixed method study design. In particular, it was required that all MSc students should be able to use the system. Hence we could not divide the student population into experimental groups. Furthermore, only students in the school were included: other disciplines and schools did not use the system. A total of 378 participants were involved in our study: 290 MSc students, 69 supervisors (academic staff), and 19 monitors (mostly postgraduate research students). 


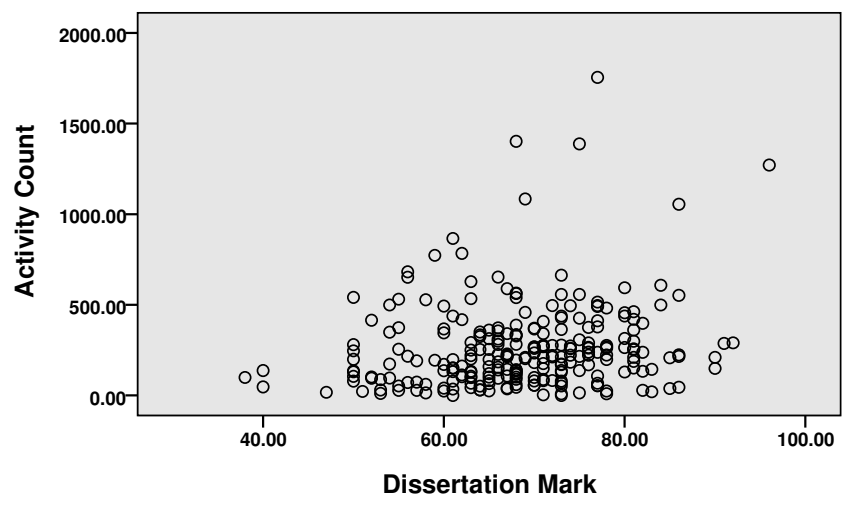

Figure 2: Scatter plot of activity count and dissertation mark

\subsection{Method}

We used a mixed method approach, whereby both quantitative and qualitative data was collected and analysed. The data comprises user activity logs on the system, progress and event attendance feedback, and email notifications sent through the system, as well as subjective data gathered using an embedded online questionnaire, launched approximately one week before the final project submission deadline. Students and their monitors were asked to take this survey in which they were to rate the motivational impact of system features, their usefulness for project management, and their agreement with several general statements. The full version of the questionnaire can be found on our website ${ }^{1}$.

\subsection{Objectives}

Our primary target was to test the effect of system use on student performance, that is, their final project mark. At this point, it is crucial to distinguish between project mark and the mark for other parts of the MSc. The former applies to the actual summer project only and is established based on the submitted dissertation, while the latter covers taught units completed before the start of the summer project, so we use the term "taught mark" for it in the remainder of this work.

Besides this primary objective, we were also interested in the effects of system use on student motivation, progress awareness, difficulty detection, user-to-user interaction, and peer support.

\section{RESULTS}

All results presented here are based on the analysis of the final data set made up by all data collected during the study. It contains 575 variables and was analysed using version 18 of the SPSS/PASW Statistics package. An outline of the types of variable used is given in Table 1.

We focussed on the main objective (see Section 3.2), that is, examining the effect of system use on student performance. System use in this context is the total number of user-system interactions. These interactions can be classified into categories, for example, the number of times a user accessed their project page, number of clicks made on the dashboard graphs, and so on. In the remainder of this paper, we shall refer to system use as the "activity count".

\footnotetext{
${ }^{1}$ http://users.ecs.soton.ac.uk/tr08r/msctrial/survey.pdf
}

Table 1: Variables in the final data set

\begin{tabular}{|l|l|}
\hline Content & Type \\
\hline Quantitative report metrics & Scale \\
\hline Qualitative report metrics & Ordinal \\
\hline Overall student progress and motivation & Ordinal \\
\hline User-system interaction counts & Scale \\
\hline Questionnaire items & Ordinal \\
\hline $\begin{array}{l}\text { Late penalties on assignments in other parts } \\
\text { of the MSc programme }\end{array}$ & Scale \\
\hline Group and project meeting count & Scale \\
\hline Score in ranking per week & Scale \\
\hline Email notification count per user & Scale \\
\hline Dissertation mark & Scale \\
\hline Mark on other parts of MSc (taught) & Scale \\
\hline Group note and reply count per user & Scale \\
\hline User-system interaction count clusters & Ordinal \\
\hline
\end{tabular}

Table 2: System activity cluster correlations

\begin{tabular}{|ll|l|l|}
\hline & & Taught Mark & Diss. Mark \\
\hline Activity Count & $r_{s}$ & 0.064 & $0.199^{* *}$ \\
& $p$ & 0.314 & 0.002 \\
\hline Cluster 1 & $r_{s}$ & 0.047 & $0.190^{* *}$ \\
& $p$ & 0.452 & 0.002 \\
\hline Cluster 2 & $r_{s}$ & 0.079 & $0.258^{* *}$ \\
& $p$ & 0.212 & 0.000 \\
\hline Cluster 3 & $r_{s}$ & 0.012 & $0.138^{*}$ \\
& $p$ & 0.856 & 0.029 \\
\hline Cluster 4 & $r_{s}$ & 0.028 & $0.170^{* *}$ \\
& $p$ & 0.656 & 0.007 \\
\hline
\end{tabular}

The most important outcome is a significant and positive correlation between students' system use and their dissertation mark $\left(r_{s}=0.199, p=0.002\right)$. This relationship is depicted in Figure 2. We used a Spearman correlation test because the activity count is not normally distributed and thus non-parametric, albeit Norman [6] suggests that parametric tests are robust enough to cope with such data. To rule out that only students who performed well before the start of their project heavily used the system, we examined the relationship between the taught mark and system activity, which is insignificant $\left(r_{s}=0.064, p=0.314\right)$. However, students with a high taught mark usually also obtained a high mark on their dissertation $\left(r_{s}=0.641, p=0.000\right)$.

We then performed a cluster analysis on the total interaction count, assigning subjects to low, medium, or high activity groups. Four different clustering algorithms were used: a cumulative activity count cluster (1), a single linkage nearest neighbour cluster (2), and two hierarchical clusters using the Ward method (3 and 4), one of them is based on Zscores. The first algorithm uses the cumulative percentage of ordered activity count values, so that users are equally distributed over the three activity groups. The result of the correlation analysis using these clusters is shown in Table 2.

Drilling down into activity categories, we found that the number of views of the main entry page (contains statistics, ranking table, and monitoring group/project breakdown), the project page, and user profile pages correlates significantly with students' dissertation mark with $r_{s}=0.216$, $r_{s}=0.202$, and $r_{s}=0.193$ at the 0.01 level $(p \leq 0.002)$, respectively. The same was found for interactions with the 
Table 3: Correlations with questionnaire items

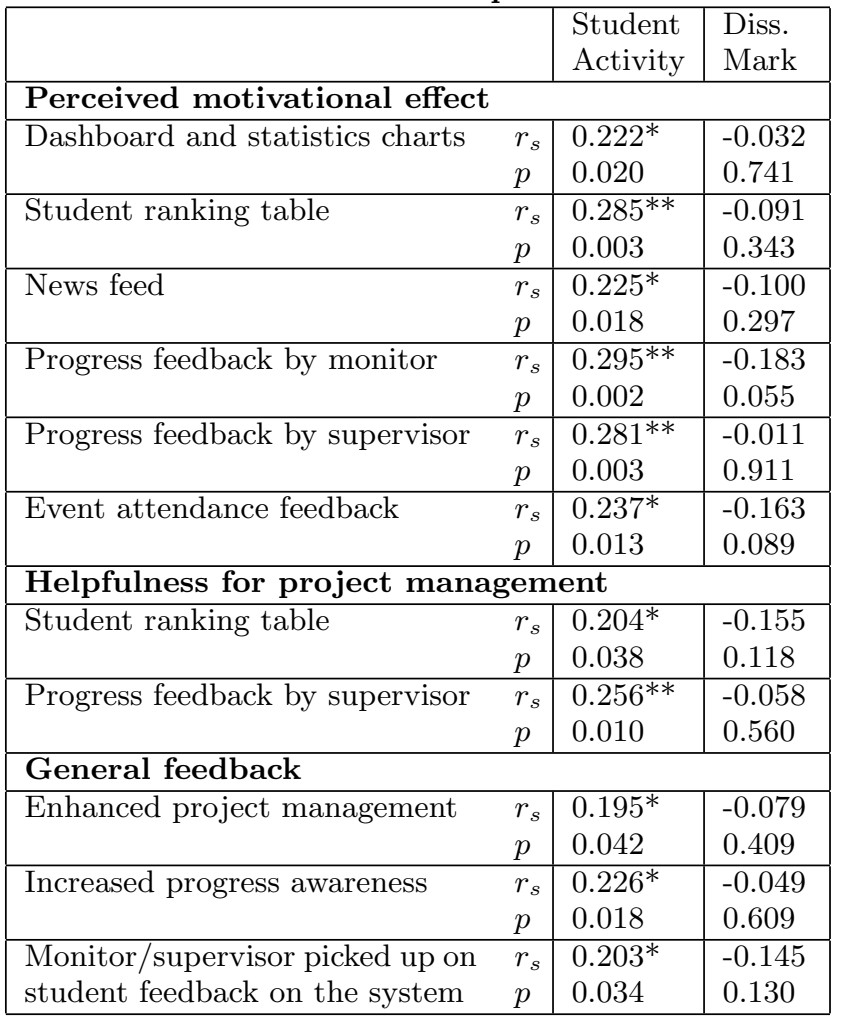

statistics dashboard on the main page and with tasks on the project page $\left(r_{s}=0.198\right.$ and $\left.r_{s}=0.153, p \leq 0.015\right)$.

We then looked at the relationship between total activity count and number of email notifications sent to students, yielding a significant and strong correlation $\left(r_{s}=0.978\right.$, $p=0.000)$. In more detail, notifications about upcoming events $\left(r_{s}=0.311, p=0.000\right)$ and other people's activity $\left(r_{s}=1.000, p=0.000\right)$ on the system had the strongest positive effect on student activity.

We were also interested in the relationship between student activity and that of their project monitor and supervisor. The Spearman correlation test yields a positive and significant relationship between students' total activity count and that of their monitor $\left(r_{s}=0.314, p=0.000\right)$. The parametric test (Pearson) also flags up a significant correlation with their supervisor's activity count $\left(r_{p}=0.168\right.$, $p=0.009$ ), but no such relationship was found using the non-parametric correlation test.

As mentioned in section 3.1, we asked students to rate the motivational effect and helpfulness of system features on a Likert scale at the end of the project. We first tested the internal consistency reliability of these items using Cronbach's alpha: motivation-related items scored 0.925 , helpfulnessrelated items 0.955 , and general statements 0.858 . The correlation analysis shows that student activity count correlates significantly with several items, as is outlined in Table 3. The table also shows that there is no significant relationship between feature ratings and dissertation mark.

Another important question was whether progress and motivation ratings submitted by students, their monitor, or their supervisor are reflected in the final dissertation mark or students' system use.
Table 4: Correlations of progress metrics

\begin{tabular}{|ll|l|l|l|}
\hline & & $\begin{array}{l}\text { Activity } \\
\text { Count }\end{array}$ & $\begin{array}{l}\text { Diss. } \\
\text { Mark }\end{array}$ & $\begin{array}{l}\text { Taught } \\
\text { Mark }\end{array}$ \\
\hline P1 & $r_{s}$ & $0.195^{* *}$ & $0.356^{* *}$ & $0.238^{* *}$ \\
& $p$ & 0.003 & 0.000 & 0.000 \\
P2 & $r_{s}$ & $0.235^{* *}$ & $0.158^{*}$ & $0.175^{*}$ \\
& $p$ & 0.001 & 0.021 & 0.011 \\
P3 & $r_{s}$ & $0.253^{* *}$ & $0.455^{* *}$ & $0.348^{* *}$ \\
& $p$ & 0.006 & 0.000 & 0.000 \\
\hline M1 & $r_{s}$ & $0.166^{*}$ & $0.322^{* *}$ & $0.226^{* *}$ \\
& $p$ & 0.011 & 0.000 & 0.000 \\
M2 & $r_{s}$ & $0.236^{* *}$ & $0.136^{*}$ & $0.147^{* *}$ \\
& $p$ & 0.001 & 0.047 & 0.032 \\
M3 & $r_{s}$ & $0.259^{* *}$ & $0.503^{* *}$ & $0.371^{* *}$ \\
& $p$ & 0.005 & 0.000 & 0.000 \\
\hline Attendance & $r_{s}$ & $0.293^{* *}$ & 0.037 & 0.101 \\
& $p$ & 0.000 & 0.598 & 0.152 \\
\hline Score & $r_{s}$ & 0.058 & $0.278^{* *}$ & $0.231^{* *}$ \\
& $p$ & 0.369 & 0.000 & 0.000 \\
\hline
\end{tabular}

Table 5: Descriptive statistics of project outcomes

\begin{tabular}{|l|r|r|r|r|}
\hline & \multicolumn{2}{|c|}{$2008 / 9$} & \multicolumn{2}{|c|}{$2009 / 10$} \\
\hline Distinction & 44 & $30.1 \%$ & 68 & $24.8 \%$ \\
\hline Pass & 92 & $63.0 \%$ & 199 & $72.6 \%$ \\
\hline Fail & 10 & $6.8 \%$ & 7 & $2.6 \%$ \\
\hline Total & 146 & & 274 & \\
\hline
\end{tabular}

For this purpose, we analysed the following metrics derived from data recorded on the system:

P1, P2, P3 Mean of self-reported, monitor-rated, and supervisor-rated overall weekly progress (value between 0 and 5)

M1, M2, M3 Mean of self-reported, monitor-rated, and supervisor-rated weekly student motivation (value between 0 and 5)

Attendance Total percentage of attended supervision and monitoring meetings

Score The mean of weekly scores in the student ranking table (includes previous metrics)

The results of the correlation analysis using these metrics is shown in Table 4. In summary, supervisors' ratings correlate most strongly with dissertation marks, while monitor feedback correlates least strongly. Surprisingly, student meeting attendance did not seem to affect marks at all.

In comparison with the previous year (2008/9), the analysis of project outcomes produces the statistics shown in Table 5. While the t-test does not show a significant difference between means $(t=0.197, p=0.844)$, a slight downward trend in student failure rate is noticeable (from $6.5 \%$ in $2008 / 9$ to $2.6 \%$ in $2009 / 10$ ).

\section{DISCUSSION}

We briefly presented the design of a web-based information system for managing and monitoring MSc summer projects, and that of a quasi-experimental mixed method study carried out in the school using this system. Our statistical results are valid for the cohort because there are significant relationships between objective and subjective data. Although 
the strength of these results is encouraging, we would have to repeat the experiment in our own and other schools and institutions to verify that they generalise.

Our primary objective was to test the system's effect on student performance. We found a significant correlation between system use and students' final project mark, however, there is no such correlation with their taught mark. However, a strong correlation exists between taught and dissertation mark, in other words, students who performed well on the taught MSc part also performed well on their summer project. The missing relationship between taught mark and system use indicates that good performers did not necessarily use the system more frequently, but those who did performed better on their project. Our finding is important in light of project work being one of the most challenging areas of any degree programme.

The analysis of system feature usage shows that the use of the progress statistics dashboard, the ranking table, the project page, and task management on that page contributed significantly to project performance. This indicates that students who were aware of their own progress compared to that of their peers and who actively managed their project using system tools performed better than those who did not. Furthermore, the role of the monitor seems to be crucial for student engagement as the correlation between their activity counts suggests. One possible explanation is that the system explicitly supports the school's monitoring scheme, and that monitors and students used the system as a platform complementing their weekly meeting routine. Also, student system use is very strongly correlated with the number of email notifications sent out by the system. This goes in line with the findings reported in [3] and [2] who argue that this is because notifications make users aware of the existence of the system and the associated users' activity.

Regarding students' subjective feedback on the motivational effect of features and their usefulness for project management, our findings support the assumption that the statistics dashboard, the news feed, and feedback submitted by monitors and supervisors are crucial features. However, motivation and helpfulness ratings correlate with system use, meaning that only those students who used the system more frequently also gave high ratings. We are currently doing more detailed research on this issue.

Finally, the results on student progress and motivation ratings suggest that supervisors were most accurate in terms of their rating's reflection on the final project mark. Obviously, students with high taught marks also gained higher motivation and progress ratings compared to those with lower taught marks, suggesting that students were able to sustain their performance during the project. Since the ratings of all three roles are significantly correlated with both marks, all ratings accurately reflect student progress and motivation. However, there is no indication that event attendance affects performance whatsoever. This finding needs to be treated with care since not all students and groups managed their meetings on the system consistently.

\subsection{Implications for System Design}

From our findings, we can compile a set of recommendations for the design of similar educational information systems. First, the full support of all staff involved is crucial. In our case, monitor activity positively influenced student activity. Supervisors were not required to use the system, but it is likely that a higher activity on their part would have had a similar effect. Second, appropriate rating systems and data visualisation of aggregated metrics and user activity improve users' progress awareness and academic performance, provided that privacy issues are taken into account. This is supported by high helpfulness and motivation ratings provided by students on these features. Finally, push notifications and simple project management tools also positively affect user activity and progress awareness.

\subsection{Future Work}

Our next step is a more detailed analysis based on these general results and a shift of focus towards our secondary claims (see Section 3.2). More specifically, we need to evaluate which factors most accurately describe the positive effect on the final project mark using principal component analysis. Furthermore, subjective feedback submitted by users together with their progress and motivation ratings needs to be coded in order to be suitable for further analysis. In particular, we are interested in whether motivating comments by monitors/supervisors influenced student progress and motivation in the following weeks.

\section{REFERENCES}

[1] B. K. Britton and A. Tesser. Effects of time-management practices on college grades. Journal of Educational Psychology, 83(33):405-410, 1991.

[2] R. Farzan, J. M. DiMicco, D. R. Millen, B. Brownholtz, W. Geyer, and C. Dugan. When the experiment is over: deploying an incentive system to all users. In Symposium on Persuasive Technology, In conjunction with the AISB 2008 Convention, 2008.

[3] A. Girgensohn and A. Lee. Making web sites be places for social interaction. In $C S C W$ '02: Proceedings of the 2002 ACM conference on Computer supported cooperative work, pages 136-145, New York, NY, USA, 2002. ACM.

[4] T. H. Macan. College students' time management: Correlations with academic performance and stress. Journal of Educational Psychology, 82(4):760-768, December 1990.

[5] T. Mochizuki, K. Yaegashi, Y. Nagamori, H. Kato, and T. Nishimori. ProBoPortable: Development of cellular phone software to prompt learners to monitor and reorganize division of labor in project-based learning. In Proceedings of EDMEDIA '08, pages 5047-5055, Chesapeake, VA, 2008. AACE.

[6] G. Norman. Likert scales, levels of measurement and the "laws" of statistics. In Advances in Health Sciences Education, volume 15, pages 625-632. Springer, 2010.

[7] T. Rebenich, A. Gravell, and T. Tiropanis. Motivating university students using a location-aware time management system with social networking features. In Proceedings of EDMEDIA '10. AACE, June 2010.

[8] T. Rebenich, A. Gravell, and T. Tiropanis. Survey of students' technology use for time management. In Proceedings of EDMEDIA '10, June 2010.

[9] J. W. Thomas. A review of research on project-based learning. Technical report, The Autodesk Foundation, 111 McInnis Parkway, San Rafael, California 94903, USA, March 2000. 